\title{
Relativistic Time Transfer for Inter-satellite Links
}

\author{
$\mathrm{Yi} \mathrm{Xie}^{1,2,3 *}$
}

${ }^{1}$ Department of Astronomy, School of Astronomy and Space Sciences, Nanjing University, Nanjing, China, ${ }^{2}$ Shanghai Key Laboratory of Space Navigation and Position Techniques, Shanghai, China, ${ }^{3}$ Key Laboratory of Modern Astronomy and Astrophysics, Nanjing University, Ministry of Education, Nanjing, China

Inter-Satellite links (ISLs) will be an important technique for a global navigation satellite system (GNSS) in the future. Based on the principles of general relativity, the time transfer in an ISL is modeled and the algorithm for onboard computation is described. It is found, in general, satellites with circular orbits and identical semi-major axes can benefit inter-satellite time transfer by canceling out terms associated with the transformations between the proper times and the Geocentric Coordinate Time. For a GPS-like GNSS, the Shapiro delay is as large as $0.1 \mathrm{~ns}$ when the ISL passes at the limb of the Earth. However, in more realistic cases, this value will decrease to about 50 ps.

Keywords: general relativity, time transfer, inter-satellite link, time comparison, global navigation satellite system

OPEN ACCESS

Edited by:

Christophe Le Poncin-Lafitte, Observatoire de Paris, France

Reviewed by:

Sven Zschocke,

Technical University Dresden,

Germany

Stefano Bertone,

University of Bern, Switzerland

*Correspondence:

YiXie

yixie@nju.edu.cn

Specialty section:

This article was submitted to

Fundamental Astronomy,

a section of the journal

Frontiers in Astronomy and Space

Sciences

Received: 03 February 2016 Accepted: 12 April 2016 Published: 26 April 2016

Citation:

Xie Y (2016) Relativistic Time Transfer for Inter-satellite Links.

Front. Astron. Space Sci. 3:15. doi: 10.3389/fspas.2016.00015

\section{INTRODUCTION}

Inter-Satellite links (ISLs), also called crosslinks, will be a promising technique for enhancing the reliability and integrity of a global navigation satellite system (GNSS). Since the early stage of the Global Positioning System (GPS), ISLs have been a part of it. ISLs are applied to transfer the Nuclear Detonation Detection System data and the autonomous navigation and ranging data between space vehicles (Sonntag, 1997). For the Block IIR satellites of GPS, the pseudorange measurements with ISLs are used by the onboard computers to update the stored navigation messages, including the ephemeris and clock states, which endows the satellites with the ability to maintain accuracy for a specified period of time after loss of contact with the control segment (Abusali et al., 1998). The network system of ISLs and its uses for the next-generation GPS was discussed (Maine et al., 2003). The European Space Agency (ESA) also assessed the potential improvements on a GNSS for navigation and dissemination performance by introducing ISLs for ranging and communication (Fernández, 2011). It was officially reportedthat China is developing and testing the new-generation Beidou Navigation Satellite System, which will have a new system of navigation signaling and ISLs.

In order to fulfill these practical purposes, time signals of onboard clocks together with other information need to be transfered among the satellites through ISLs for ranging and computing clock offsets. In the procedure of time transfer, Einstein's general relativity (GR) has "long since passed from the realm of theoretical physics to the realm of engineering design" (Nelson, 2011). In fact, GR is an inevitable part of a GNSS (Ashby, 2003; Han et al., 2011). In the light of the principles of GR, it is necessary to abandon the Newton's absolute time for different kinds of times: proper times and coordinate times (Misner et al., 1973; Landau and Lifshitz, 1975).

Theoretically, the readings of an ideal clock give the proper time $\tau$, which is an observable and only belongs to the clock itself. However, there exists no such thing as an ideal clock. An atomic clock, which is widely used on the ground and in space, drifts from the ideal clock with some

\footnotetext{
$\overline{{ }^{1} \text { http://www.beidou.gov.cn/2015/04/01/20150401 }}$ b4b91ddc213a45129a665ea3272b5aed.html.
} 
known and unknown factors. The coordinate times cannot be measured directly. However, they might be used as variables in the equations of motion of celestial and artificial bodies and light rays. The coordinate times are connected with the proper time through the four-dimensional spacetime interval, which changes the way of clock synchronization and time transfer significantly (Petit and Wolf, 2005; Nelson, 2007, 2011). Experiments involving time/frequency transfer might also be used for testing theories of gravity (Samain, 2002; Cacciapuoti and Salomon, 2009; Christophe et al., 2009; Wolf et al., 2009; Christophe et al., 2012; Deng and Xie, 2013a,b, 2014; Hees et al., 2014; Zhang et al., 2014; Delva et al., 2015; Xie and Huang, 2015).

The relativistic time transfers in various contexts, such as in the vicinity of the Earth (Klioner, 1992; Petit and Wolf, 1994; Wolf and Petit, 1995; Petit and Wolf, 1997; Kouba, 2002, 2004; Petit and Wolf, 2005; Nelson, 2007, 2011) and in the Solar System (Nelson, 2007, 2011; Deng, 2012; Pan and Xie, 2013, 2014, 2015), have been intensively studied and discussed. In such contexts, one clock is on-board a space vehicle and the other clock or a user is usually on the ground. However, for ISLs, each end of a link is a satellite with clocks in orbital motion, which makes the description of the time of light propagation in the ISL different from previously mentioned cases. In the present work, we will model the time transfer through ISLs and discuss its algorithm for onboard computation, where the relativistic effects are fully taken into account.

The paper is organized as follows. Section 2 is devoted to modeling the time transfer through ISLs. Some mathematical details for this purpose can be found in the Appendix. The algorithm for onboard computation is also discussed. Finally, in Section 3, we summarize our results and discuss their implication.

\section{GENERAL RELATIVISTIC DESCRIPTION OF TIME TRANSFER IN AN ISL}

We consider an ISL between two satellites 1 and 2 in the vicinity of the Earth. It is also assumed that these two satellites belong to a GNSS like GPS. The proper times of their onboard clocks are $\tau_{1}$ and $\tau_{2}$. Satellite 2 emits electromagnetic signals encoded with its proper time of this emission of $\tau_{2}$ and other necessary information, such as its ephemeris and clock offset. Satellite 1 receives these signals at its proper time $\tau_{1}$, decodes out $\tau_{2}$ and other messages and computes $\tau_{1}-\tau_{2}$. In the framework of the International Astronomical Union (IAU) 2000 Resolutions for general relativistic reference systems (Soffel et al., 2003), we describe this problem in the Geocentric Celestial Reference System (GCRS), which is physically adequate to describe processes occurring in the vicinity of Earth. For the time transfer in the ISL, we can have

$$
\frac{\mathrm{d} \tau_{1}}{\mathrm{~d} \tau_{2}}=\frac{\mathrm{d} \tau_{1}}{\mathrm{~d} T_{1}} \frac{\mathrm{d} T_{1}}{\mathrm{~d} T_{2}} \frac{\mathrm{d} T_{2}}{\mathrm{~d} \tau_{2}},
$$

where $T_{1}$ and $T_{2}$ are the time coordinates of GCRS, called the Geocentric Coordinate Time (TCG).
The first and third terms on the r.h.s of Equation (1) account for the relativistic four-dimensional transformations between the proper time and TCG. Considering a post-Newtonian development and keeping all terms larger than one part in $10^{18}$, the first term on the r.h.s of Equation (1) reads (Wolf and Petit, 1995; Petit and Wolf, 1997)

$$
\begin{aligned}
\frac{\mathrm{d} \tau_{1}}{\mathrm{~d} T_{1}}= & 1-\epsilon^{2}\left[U_{\mathrm{E}}\left(\boldsymbol{X}_{1}\right)+\frac{1}{2} \boldsymbol{V}_{1}^{2}+\bar{U}\left(\boldsymbol{x}_{\mathrm{E}}+\boldsymbol{X}_{1}\right)-\bar{U}_{\mathrm{E}}\left(\boldsymbol{x}_{\mathrm{E}}\right)\right. \\
& \left.-\boldsymbol{X}_{1} \cdot \nabla \bar{U}\left(\boldsymbol{x}_{\mathrm{E}}\right)+\boldsymbol{X}_{1} \cdot \boldsymbol{Q}\right]+\mathcal{O}\left(\epsilon^{4}\right) .
\end{aligned}
$$

Here, $\epsilon=c^{-1}$ and $c$ is the speed of light; $U_{\mathrm{E}}\left(\boldsymbol{X}_{1}\right)$ is the Newtonian gravitational potential of the Earth evaluated at the position of satellite $1, \boldsymbol{X}_{1} ; \boldsymbol{V}_{1}$ is the velocity of satellite 1 in GCRS; $\bar{U}(\boldsymbol{x})$ is the Newtonian gravitational potential of external masses (except the Earth) evaluated at the coordinate of $\boldsymbol{x} ; \boldsymbol{x}_{\mathrm{E}}$ is the coordinate of the Earth's center of mass in the Solar System Barycentric Celestial Reference System (BCRS); the $\boldsymbol{Q}$ term is related to the four-acceleration of the Earth's center of mass in the external gravitational field due to its mass quadrupole and it is numerically at the order of $\sim 10^{-11} \mathrm{~m} \mathrm{~s}^{-2}$. It was found in Kouba (2004) that, at the pico-second (ps) precision level (or the inaccuracy of frequency of $10^{-16}$ ), the effects of external masses can be neglected in time transfer for GPS. Therefore, we can simplify Equation (2) as

$$
\frac{\mathrm{d} \tau_{1}}{\mathrm{~d} T_{1}}=1-\epsilon^{2}\left[U_{\mathrm{E}}\left(\boldsymbol{X}_{1}\right)+\frac{1}{2} \boldsymbol{V}_{1}^{2}\right]+\mathcal{O}\left(\epsilon^{4}\right),
$$

and we get a similar equation for the third term in Equation (1) as

$$
\frac{\mathrm{d} T_{2}}{\mathrm{~d} \tau_{2}}=1+\epsilon^{2}\left[U_{\mathrm{E}}\left(\boldsymbol{X}_{2}\right)+\frac{1}{2} \boldsymbol{V}_{2}^{2}\right]+\mathcal{O}\left(\epsilon^{4}\right) .
$$

It was also pointed out in Kouba (2004) that only the first oblateness term $J_{2}$ in $U_{\mathrm{E}}$ is necessary for the orbital altitude of GPS satellites. In the GPS relativistic transformations between the proper time and TCG, both the orbit perturbations and the Earth gravity field oblateness $J_{2}$, which is a main cause of the perturbations, need to be taken into account together (Kouba, 2004).

The second term in Equation (1) describes the propagation of the electromagnetic signal from satellite 2 to satellite 1 in TCG. For a one-way signal transmission, we can have the coordinate time interval as (Blanchet et al., 2001)

$$
T_{1}-T_{2}=\epsilon F_{1}\left(T_{1}, T_{2}\right)+\epsilon^{3} F_{2}\left(T_{1}, T_{2}\right)+\mathcal{O}\left(\epsilon^{4}\right),
$$

where

$$
\begin{aligned}
& F_{1}\left(T_{1}, T_{2}\right)=\left|\boldsymbol{X}_{1}\left(T_{1}\right)-\boldsymbol{X}_{2}\left(T_{2}\right)\right|, \\
& F_{2}\left(T_{1}, T_{2}\right)=2 G M_{\mathrm{E}} \ln \left[\frac{R\left(T_{1}\right)+\boldsymbol{X}\left(T_{1}\right) \cdot \hat{\boldsymbol{N}}}{R\left(T_{2}\right)+\boldsymbol{X}\left(T_{2}\right) \cdot \hat{\boldsymbol{N}}}\right] .
\end{aligned}
$$

Here, $\epsilon F_{1}$ is the Euclidean geometric effect and $\epsilon^{3} F_{2}$ is the Shapiro time delay (Shapiro, 1964; Weinberg, 1972) where we 
only consider the monopole component of the Earth's potential. $R, X$, and $\hat{N}$ are quantities associated with the propagation of the signal so that

$$
\begin{aligned}
\hat{\boldsymbol{N}} & =\frac{\boldsymbol{X}_{1}-\boldsymbol{X}_{2}}{\left|\boldsymbol{X}_{1}-\boldsymbol{X}_{2}\right|}, \\
\boldsymbol{X}(T) & =\boldsymbol{X}_{2}\left(T_{2}\right)+c \hat{\boldsymbol{N}}\left(T-T_{2}\right)+\mathcal{O}\left(\epsilon^{2}\right), \\
R(T) & =|\boldsymbol{X}(T)| .
\end{aligned}
$$

Substituting Equations (3-5) into Equation (1), we can have

$$
\begin{aligned}
\frac{\mathrm{d} \tau_{1}}{\mathrm{~d} \tau_{2}}= & 1+\epsilon \frac{\mathrm{d} F_{1}}{\mathrm{~d} T_{2}}-\epsilon^{2}\left[U_{\mathrm{E}}\left(\boldsymbol{X}_{1}\right)+\frac{1}{2} \boldsymbol{V}_{1}^{2}\right]+\epsilon^{2}\left[U_{\mathrm{E}}\left(\boldsymbol{X}_{2}\right)+\frac{1}{2} \boldsymbol{V}_{2}^{2}\right] \\
& +\epsilon^{3} \frac{\mathrm{d} F_{2}}{\mathrm{~d} T_{2}}-\epsilon^{3} \frac{\mathrm{d} F_{1}}{\mathrm{~d} T_{2}}\left[U_{\mathrm{E}}\left(\boldsymbol{X}_{1}\right)+\frac{1}{2} \boldsymbol{V}_{1}^{2}\right] \\
& +\epsilon^{3} \frac{\mathrm{d} F_{1}}{\mathrm{~d} T_{2}}\left[U_{\mathrm{E}}\left(\boldsymbol{X}_{2}\right)+\frac{1}{2} \boldsymbol{V}_{2}^{2}\right]+\mathcal{O}\left(\epsilon^{4}\right) .
\end{aligned}
$$

Leaving the details of integration in the Appendix, we can finally have

$$
\tau_{1}-\tau_{2}=\Delta \tau_{\mathrm{E}}+\Delta \tau_{\mathrm{S}_{1}}+\Delta \tau_{\mathrm{S}_{2}}+\Delta \tau_{\mathrm{Sh}}+\mathcal{O}\left(\epsilon^{4}\right)
$$

where the terms due to Euclidean geometry, $\Delta \tau_{\mathrm{E}}$, time transformations for satellite 1 and $2, \Delta \tau_{S_{1}}$ and $\Delta \tau_{S_{2}}$, and the Shapiro delay, $\Delta \tau_{\mathrm{Sh}}$, are

$$
\begin{aligned}
\Delta \tau_{\mathrm{E}} & =\epsilon\left|\boldsymbol{X}_{1}\left(T_{1}\right)-\boldsymbol{X}_{2}\left(T_{2}\right)\right|, \\
\Delta \tau_{\mathrm{S}_{1}} & =-\epsilon^{2} \int_{\tau_{2}}^{\tau_{1}}\left\{U_{\mathrm{E}}\left[\boldsymbol{X}_{1}(\tau)\right]+\frac{1}{2} \boldsymbol{V}_{1}^{2}(\tau)\right\} \mathrm{d} \tau, \\
\Delta \tau_{\mathrm{S}_{2}} & =\epsilon^{2} \int_{\tau_{2}}^{\tau_{1}}\left\{U_{\mathrm{E}}\left[\boldsymbol{X}_{2}(\tau)\right]+\frac{1}{2} \boldsymbol{V}_{2}^{2}(\tau)\right\} \mathrm{d} \tau, \\
\Delta \tau_{\mathrm{Sh}} & =2 \epsilon^{3} G M_{\mathrm{E}} \ln \left[\frac{\left|\boldsymbol{X}_{1}\left(T_{1}\right)\right|+\left|\boldsymbol{X}_{2}\left(T_{2}\right)\right|+\left|\boldsymbol{X}_{1}\left(T_{1}\right)-\boldsymbol{X}_{2}\left(T_{2}\right)\right|}{\left|\boldsymbol{X}_{1}\left(T_{1}\right)\right|+\left|\boldsymbol{X}_{2}\left(T_{2}\right)\right|-\left|\boldsymbol{X}_{1}\left(T_{1}\right)-\boldsymbol{X}_{2}\left(T_{2}\right)\right|}\right] .
\end{aligned}
$$

In order to calculate all of the above terms, models of clock offsets and ephemerides for both satellites are required. In principle, according to the model of clock offset used in GNSS data processing (Ashby and Spilker, 1996), the difference between the proper time $\tau$ and $T$ in TCG can be determined as

$$
T-\tau=c_{0}+c_{1}\left(\tau-\tau_{0}\right)+c_{2}\left(\tau-\tau_{0}\right)^{2}+2 \sqrt{G M_{\mathrm{E}} a} e \sin E,
$$

where $c_{0}, c_{1}$, and $c_{2}$ are the coefficients of the clock offset model, $a$ is the orbital semi-major axis of a satellite, $e$ is the orbital eccentricity and $E$ is the eccentric anomaly. All these quantities are available in the navigation messages.

For the Euclidean term $\Delta \tau_{\mathrm{E}}, T_{1}$, and $T_{2}$ can be obtained by the clock offset models by Equation (17), and $\boldsymbol{X}_{1}\left(T_{1}\right)$ and $\boldsymbol{X}_{2}\left(T_{2}\right)$ can be calculated by the ephemerides, which are basically represented in the form of orbital elements and are also available in the navigation messages. If we consider a GPS-like GNSS that $a_{1} \approx a_{2} \approx a$ and $e_{1} \approx e_{2} \approx 0$, where $a_{1}$ and $a_{2}$ are the orbital semi-major axes of satellite 1 and $2, e_{1}$ and $e_{2}$ are the orbital eccentricities and $a \approx 26559.7 \mathrm{~km}$, we can find that $\left|\boldsymbol{X}_{1}\left(T_{1}\right)\right| \approx\left|\boldsymbol{X}_{2}\left(T_{2}\right)\right| \approx a,\left|\boldsymbol{X}_{1}\left(T_{1}\right)-\boldsymbol{X}_{2}\left(T_{2}\right)\right| \lesssim 2 \sqrt{\boldsymbol{a}^{2}-R_{\oplus}^{2}}$ and $\Delta \tau_{\mathrm{E}} \lesssim 0.172 \mathrm{~s}$, where $R_{\oplus}$ is the radius of the Earth. In order to maximize $\left|\boldsymbol{X}_{1}\left(T_{1}\right)-\boldsymbol{X}_{2}\left(T_{2}\right)\right|$, the ISL needs to pass through the Earth, which never happens in practice. In a more realistic situation, when $\boldsymbol{X}_{1}\left(T_{1}\right)$ is perpendicular to $\boldsymbol{X}_{2}\left(T_{2}\right), \Delta \tau_{\mathrm{E}}$ is about $0.125 \mathrm{~s}$.

For the time transformation terms, when the oblateness of the Earth $J_{2}$ can be neglected, it can be found that (Nelson, 2011)

$$
\begin{aligned}
\Delta \tau_{\mathrm{S}_{1}}= & -\epsilon^{2}\left(\frac{3}{2} \frac{G M_{\mathrm{E}}}{a_{1}} \Delta \tau+\left.2 \sqrt{G M_{\mathrm{E}} a_{1}} e_{1} \sin E_{1}\right|_{T_{2}} ^{T_{1}}\right) \\
& +\mathcal{O}\left(\epsilon^{2} J_{2}\right) \\
\Delta \tau_{\mathrm{S}_{2}}= & \epsilon^{2}\left(\frac{3}{2} \frac{G M_{\mathrm{E}}}{a_{2}} \Delta \tau+\left.2 \sqrt{G M_{\mathrm{E}} a_{2}} e_{2} \sin E_{2}\right|_{T_{2}} ^{T_{1}}\right) \\
& +\mathcal{O}\left(\epsilon^{2} J_{2}\right)
\end{aligned}
$$

where $\Delta \tau=\tau_{1}-\tau_{2}$ and $E_{1}$ and $E_{2}$ are the eccentric anomalies of the two satellites. When satellite 1 and 2 are set in the orbits with almost the same size and close to circles, $\Delta \tau_{\mathrm{S}_{1}}+\Delta \tau_{\mathrm{S}_{2}}$ will nearly cancel out each other. In the case that $J_{2}$ has to be taken into account, additional corrections will be needed and their mathematical expressions can be found in Ashby (2003); Kouba (2004); Nelson (2011). For a GPS satellite with an inclination of $i=55.0^{\circ}$, the drift rate of the secular term due to $J_{2}$ is at the level of $-3.4 \times 10^{-17}$ and the amplitude of the periodic effect is 24 ps. For a GPS-like system but with different inclinations, the maximum value of the drift rate is about $5.2 \times 10^{-15}$ for $i=0^{\circ}$ and the maximum value of the amplitude of the periodic effect is 36 ps for $i=90^{\circ}$. Because of its dependence of the orbital inclination, the argument of perigee and the true anomaly, the $J_{2}$-associated component in $\Delta \tau_{\mathrm{S}_{1}}+\Delta \tau_{\mathrm{S}_{2}}$ will generally not cancel out each other.

For the Shapiro time delay, if we consider that $a_{1} \approx a_{2} \approx a$ and $e_{1} \approx e_{2} \approx 0$, we can find that the maximum value of $\Delta \tau_{\mathrm{Sh}}=0.12 \mathrm{~ns}$ when the ISL is passing the limb of the Earth. However, in more probable cases, this value will be much smaller. For example, when $\boldsymbol{X}_{1}\left(T_{1}\right)$ is perpendicular to $\boldsymbol{X}_{2}\left(T_{2}\right), \Delta \tau_{\mathrm{Sh}}$ is about 52 ps.

It was reported in Hadas and Bosy (2015) that, for GPS, realtime clocks accuracy is $0.28 \mathrm{~ns}$ and orbits accuracy is $5 \mathrm{~cm}(0.17$ $n s)$. Based on the above estimation, the $J_{2}$ effects and the Shapiro time delay can be neglected for ISLs in a GPS-like system.

\section{CONCLUSIONS AND DISCUSSION}

In the framework of GR, we investigate the time transfer for ISLs, which will be a promising technique for a GNSS in the future. We give a model describing the difference between two onboard clocks of an ISL and discuss its algorithm for onboard computation. It is found that the difference between the proper times of two satellites contains the contributions from Euclidean geometry, time transformations between the proper time and TCG for these satellites and the Shapiro delay. For a GPS-like system, the net effect of the time transformations 
is close to 0 because they cancel out each other and the $J_{2}$ effects in these transformations are less than several tens of ps. The Shapiro delay is about 52 ps in such a system. For current clocks accuracy of GPS, the effects of $J_{2}$ and the Shapiro delay can be neglected. After all of the differences among the clocks in a GNSS are obtained, the ephemeris and the clock states can be evaluated and updated to maintain the system.

In practice, some additional factors need to be taken into account as well, such as the time delay caused by the Earth's atmosphere and instruments. Due to the limited capability of on-board computation, the question about how to optimize the constellation of a GNSS and the measurements through ISLs is another important issue. More detailed and specific case studies are definitely required.

In the future, when optical clocks and laser links (Chou et al., 2010a; Chou et al., 2010b; Predehl et al., 2012; Bloom et al., 2014) are deployed and applied in space, the external gravitational effLects can no longer be neglected and will have

\section{REFERENCES}

Abusali, P. A. M., Tapley, B. D., and Schutz, B. E. (1998). Autonomous navigation of global positioning system satellites using cross-link measurements. J. Guid. Control Dynam. 21, 321-327. doi: 10.2514/2.4238

Aoyama, S., Tazai, R., and Ichiki, K. (2014). Upper limit on the amplitude of gravitational waves around $0.1 \mathrm{~Hz}$ from the Global Positioning System. Phys. Rev. D 89:067101. doi: 10.1103/PhysRevD.89.067101

Ashby, N. (2003). Relativity in the global positioning system. Living Rev. Relativ. 6, 1. doi: 10.12942/lrr-2003-1. Available online at: http://relativity.livingreviews. org/Articles/lrr-2003-1/

Ashby, N., and Spilker, J. (1996). "Introduction to relativistic effects on the global positioning system," in Global Positioning System: Theory and Applications, Vol. 1, eds B. Parkinson, J. Spilker Jr., P. Axelrad, and P. Enge (Reston, VA: American Institute of Aeronautics and Astronautics), 623-697.

Bertolami, O., and Páramos, J. (2011). Using global positioning systems to test extensions of general relativity. Int. J. Mod. Phys. D 20, 1617-1641. doi: 10.1142/S0218271811019451

Blanchet, L., Salomon, C., Teyssandier, P., and Wolf, P. (2001). Relativistic theory for time and frequency transfer to order $\mathrm{c}^{-3}$. Astron. Astrophys. 370, 320-329. doi: 10.1051/0004-6361:20010233

Bloom, B. J., Nicholson, T. L., Williams, J. R., Campbell, S. L., Bishof, M., Zhang, X., et al. (2014). An optical lattice clock with accuracy and stability at the $10^{-18}$ level. Nature 506, 71-75. doi: 10.1038/nature12941

Cacciapuoti, L., and Salomon, C. (2009). Space clocks and fundamental tests: the ACES experiment. Eur. Phys. J. Spec. Top. 172, 57-68. doi: 10.1140/epjst/e200901041-7

Chou, C. W., Hume, D. B., Koelemeij, J. C. J., Wineland, D. J., and Rosenband, T. (2010a). Frequency comparison of two high-accuracy al ${ }^{+}$optical clocks. Phys. Rev. Lett. 104:070802. doi: 10.1103/PhysRevLett.104.070802

Chou, C. W., Hume, D. B., Rosenband, T., and Wineland, D. J. (2010b). Optical clocks and relativity. Science 329, 1630. doi: 10.1126/science.1192720

Christophe, B., Andersen, P. H., Anderson, J. D., Asmar, S., Bério, P., Bertolami, O., et al. (2009). Odyssey: a solar system mission. Exp. Astron. 23, 529-547. doi: 10.1007/s10686-008-9084-y

Christophe, B., Spilker, L. J., Anderson, J. D., André, N., Asmar, S. W., Aurnou, J., et al. (2012). OSS (Outer Solar System): a fundamental and planetary physics mission to Neptune, Triton and the Kuiper Belt. Exp. Astron. 34, 203-242. doi: 10.1007/s10686-012-9309-y

Delva, P., Hees, A., Bertone, S., Richard, E., and Wolf, P. (2015). Test of the gravitational redshift with stable clocks in eccentric orbits: application to to be carefully included (Blanchet et al., 2001; Soffel et al., 2003; Petit and Luzum, 2010). Moreover, an accurate processing of ISL data might also offer a new opportunity for the application of GNSS in fundamental physics and astronomy (Wolf and Petit, 1997; Bertolami and Páramos, 2011; Kentosh and Mohageg, 2012; Aoyama et al., 2014).

\section{AUTHOR CONTRIBUTIONS}

The author confirms being the sole contributor of this work and approved it for publication.

\section{ACKNOWLEDGMENTS}

I acknowledge the helpful comments and suggestions from my referees. The work is funded by the National Natural Science Foundation of China under Grant No. 11573015 and the Opening Project of Shanghai Key Laboratory of Space Navigation and Position Techniques under Grant No. 14DZ2276100.

Galileo satellites 5 and 6. Class. Quant. Grav. 32:232003. doi: 10.1088/0264$9381 / 32 / 23 / 232003$

Deng, X.-M. (2012). The transformation between $\tau$ and TCB for deep space missions under IAU resolutions. Res. Astron. Astrophys. 11, 703-712. doi: 10.1088/1674-4527/12/6/010

Deng, X.-M., and Xie, Y. (2013a). The effect of $f(T)$ gravity on an interplanetary clock and its time transfer link. Res. Astron. Astrophys. 13, 1225-1230. doi: 10.1088/1674-4527/13/10/010

Deng, X.-M., and Xie, Y. (2013b). Yukawa effects on the clock onboard a dragfree satellite. Mon. Not. R. Astron. Soc. 431, 3236-3239. doi: 10.1093/mnras/s $\mathrm{tt} 404$

Deng, X.-M., and Xie, Y. (2014). Spacecraft Doppler tracking with possible violations of LLI and LPI: a theoretical modeling. Res. Astron. Astrophys. 14:319. doi: 10.1088/1674-4527/14/3/006

Fernández, F. A. (2011). Inter-satellite ranging and inter-satellite communication links for enhancing GNSS satellite broadcast navigation data. Adv. Space Res. 47, 786-801. doi: 10.1016/j.asr.2010.10.002

Hadas, T., and Bosy, J. (2015). IGS RTS precise orbits and clocks verification and quality degradation over time. GPS Solut. 19, 93-105. doi: 10.1007/s10291-0140369-5

Han, C., Yang, Y., and Cai, Z. (2011). BeiDou navigation satellite system and its time scales. Metrologia 48, S213-S218. doi: 10.1088/0026-1394/48/4/S13

Hees, A., Folkner, W. M., Jacobson, R. A., and Park, R. S. (2014). Constraints on modified Newtonian dynamics theories from radio tracking data of the Cassini spacecraft. Phys. Rev. D 89:102002. doi: 10.1103/PhysRevD.89.102002

Kentosh, J., and Mohageg, M. (2012). Global positioning system test of the local position invariance of Planck's constant. Phys. Rev. Lett. 108:110801. doi: 10.1103/PhysRevLett.108.110801

Klioner, S. A. (1992). The problem of clock synchronization - a relativistic approach. Celest. Mech. Dyn. Astron. 53, 81-109. doi: 10.1007/BF00049363

Kouba, J. (2002). Relativistic time transformations in gps. GPS Solut. 5, 1-9. doi: $10.1007 /$ PL00012907

Kouba, J. (2004). Improved relativistic transformations in gps. GPS Solut. 8, 170-180. doi: 10.1007/s10291-004-0102-x

Landau, L. D., and Lifshitz, E. M. (1975). The Classical Theory of Fields. Oxford: Pergamon Press.

Maine, K. P., Anderson, P., and Langer, J. (2003). "Crosslinks for the nextgeneration GPS," in Aerospace Conference, 2003. Proceedings. 2003 IEEE, Vol. 4 (Big Sky, MT), 1589-1596.

Misner, C. W., Thorne, K. S., and Wheeler, J. A. (1973). Gravitation. San Francisco, CA: Freeman. 
Nelson, R. A. (2007). "Relativistic time transfer in the solar system," in Frequency Control Symposium, 2007 Joint with the 21st European Frequency and Time Forum. IEEE International (Geneva), 1278-1283.

Nelson, R. A. (2011). Relativistic time transfer in the vicinity of the Earth and in the Solar System. Metrologia 48, S171-S180. doi: 10.1088/0026-1394/ $48 / 4 / 507$

Pan, J.-Y., and Xie, Y. (2013). Relativistic transformation between $\tau$ and TCB for Mars missions: Fourier analysis on its accessibility with clock offset. Res. Astron. Astrophys. 13, 1358-1362. doi: 10.1088/1674-4527/ $13 / 11 / 006$

Pan, J.-Y., and Xie, Y. (2014). Relativistic transformation between $\tau$ and TCG for Mars missions under IAU Resolutions. Res. Astron. Astrophys. 14, 233-240. doi: $10.1088 / 1674-4527 / 14 / 2 / 011$

Pan, J.-Y., and Xie, Y. (2015). Relativistic algorithm for time transfer in Mars missions under IAU Resolutions: an analytic approach. Res. Astron. Astrophys. 15, 281-292. doi: 10.1088/1674-4527/15/2/011

Petit, G., and Luzum, B. (eds.). (2010). IERS Conventions (2010), IERS Technical Note; 36. Bundesamt für Kartographie und Geodäsie.

Petit, G., and Wolf, P. (1994). Relativistic theory for picosecond time transfer in the vicinity of the earth. Astron. Astrophys. 286, 971-977.

Petit, G., and Wolf, P. (1997). Computation of the relativistic rate shift of a frequency standard. IEEE Trans. Instrum. Meas. 46, 201-204.

Petit, G., and Wolf, P. (2005). Relativistic theory for time comparisons: a review. Metrologia 42:138. doi: 10.1088/0026-1394/42/3/S14

Predehl, K., Grosche, G., Raupach, S. M. F., Droste, S., Terra, O., Alnis, J., et al. (2012). A 920-kilometer optical fiber link for frequency metrology at the 19th decimal place. Science 336:441. doi: 10.1126/science. 1218442

Samain, E. (2002). "One way laser ranging in the solar system : TIPO," in EGS General Assembly Conference Abstracts, Vol. 27 of EGS General Assembly Conference Abstracts (Nice), 5808.

Shapiro, I. I. (1964). Fourth test of general relativity. Phys. Rev. Lett. 13, 789-791. doi: 10.1103/PhysRevLett.13.789

Soffel, M., Klioner, S. A., Petit, G., Wolf, P., Kopeikin, S. M., Bretagnon, P., et al. (2003). The IAU 2000 resolutions for astrometry, celestial mechanics, and metrology in the relativistic framework: explanatory supplement. Astron. J. 126, 2687-2706. doi: $10.1086 / 378162$
Sonntag, H. E. (1997). “Block IIR UHF crosslink enhancements study," in Proceedings of the 1997 National Technical Meeting of The Institute of Navigation (Santa Monica, CA), 819-828.

Weinberg, S. (1972). Gravitation and Cosmology: Principles and Applications of the General Theory of Relativity. New York, NY: Wiley.

Wolf, P., Bordé, C. J., Clairon, A., Duchayne, L., Landragin, A., Lemonde, P., et al. (2009). Quantum physics exploring gravity in the outer solar system: the SAGAS project. Exp. Astron. 23, 651-687. doi: 10.1007/s10686-008-9 118-5

Wolf, P., and Petit, G. (1995). Relativistic theory for clock syntonization and the realization of geocentric coordinate times. Astron. Astrophys. 30 4:653.

Wolf, P., and Petit, G. (1997). Satellite test of special relativity using the global positioning system. Phys. Rev. A 56, 4405-4409.

Xie, Y., and Huang, Y. (2015). Spacecraft Doppler tracking with possible violations of LLI and LPI: upper bounds from one-way measurements on MEX. Res. Astron. Astrophys. 15:1751. doi: 10.1088/1674-4527/15/1 $0 / 012$

Zhang, Y.-F., Zhang, X.-Z., Liu, J.-H., Huang, Y., and Xie, Y. (2014). Spacecraft Doppler tracking with possible violations of LLI and LPI: preliminary bounds on LLI from Mars Express. Res. Astron. Astrophys. 14, 1201-1206. doi: $10.1088 / 1674-4527 / 14 / 9 / 010$

Conflict of Interest Statement: The author declares that the research was conducted in the absence of any commercial or financial relationships that could be construed as a potential conflict of interest.

The Editorial Office is aware of co-publications from the Associate Editor and Reviewer SB, and confirms the review was carried out to the highest degree of professionalism, and is free of bias.

Copyright (๑) 2016 Xie. This is an open-access article distributed under the terms of the Creative Commons Attribution License (CC BY). The use, distribution or reproduction in other forums is permitted, provided the original author $(s)$ or licensor are credited and that the original publication in this journal is cited, in accordance with accepted academic practice. No use, distribution or reproduction is permitted which does not comply with these terms. 


\section{APPENDIX: DERIVATION OF EQUATION}

\section{(12)}

Integrating Equation (11), we can have

$$
\begin{aligned}
\tau_{1}-\tau_{2}= & \epsilon \int \frac{\mathrm{d} F_{1}}{\mathrm{~d} T_{2}} \mathrm{~d} \tau_{2}-\epsilon^{2} \int\left[U_{\mathrm{E}}\left(\boldsymbol{X}_{1}\right)+\frac{1}{2} \boldsymbol{V}_{1}^{2}\right] \mathrm{d} \tau_{2} \\
& +\epsilon^{2} \int\left[U_{\mathrm{E}}\left(\boldsymbol{X}_{2}\right)+\frac{1}{2} \boldsymbol{V}_{2}^{2}\right] \mathrm{d} \tau_{2} \\
& -\epsilon^{3} \int \frac{\mathrm{d} F_{1}}{\mathrm{~d} T_{2}}\left[U_{\mathrm{E}}\left(\boldsymbol{X}_{1}\right)+\frac{1}{2} \boldsymbol{V}_{1}^{2}\right] \mathrm{d} \tau_{2} \\
& +\epsilon^{3} \int \frac{\mathrm{d} F_{1}}{\mathrm{~d} T_{2}}\left[U_{\mathrm{E}}\left(\boldsymbol{X}_{2}\right)+\frac{1}{2} \boldsymbol{V}_{2}^{2}\right] \mathrm{d} \tau_{2} \\
& +\epsilon^{3} \int \frac{\mathrm{d} F_{2}}{\mathrm{~d} T_{2}} \mathrm{~d} \tau_{2}+\mathcal{O}\left(\epsilon^{4}\right) .
\end{aligned}
$$

Since Equation (4) can lead to

$$
\mathrm{d} \tau_{2}=\left\{1-\epsilon^{2}\left[U_{\mathrm{E}}\left(\boldsymbol{X}_{2}\right)+\frac{1}{2} \boldsymbol{V}_{2}^{2}\right]\right\} \mathrm{d} T_{2}+\mathcal{O}\left(\epsilon^{4}\right)
$$

and Equation (5) can give

$$
\frac{\mathrm{d} T_{1}}{\mathrm{~d} T_{2}}=1+\epsilon \frac{\mathrm{d} F_{1}}{\mathrm{~d} T_{2}}+\epsilon^{3} \frac{\mathrm{d} F_{2}}{\mathrm{~d} T_{2}}+\mathcal{O}\left(\epsilon^{4}\right),
$$

we can combine some terms in Equation (A1) as

$$
\begin{aligned}
\epsilon \int \frac{\mathrm{d} F_{1}}{\mathrm{~d} T_{2}} \mathrm{~d} \tau_{2}+ & \epsilon^{3} \int \frac{\mathrm{d} F_{1}}{\mathrm{~d} T_{2}}\left[U_{\mathrm{E}}\left(\boldsymbol{X}_{2}\right)+\frac{1}{2} \boldsymbol{V}_{2}^{2}\right] \mathrm{d} \tau_{2} \\
& =\epsilon\left|\boldsymbol{X}_{1}\left(T_{1}\right)-\boldsymbol{X}_{2}\left(T_{2}\right)\right|+\mathcal{O}\left(\epsilon^{4}\right)
\end{aligned}
$$

$$
\begin{aligned}
& -\epsilon^{2} \int\left[U_{\mathrm{E}}\left(\boldsymbol{X}_{1}\right)+\frac{1}{2} \boldsymbol{V}_{1}^{2}\right] \mathrm{d} \tau_{2}-\epsilon^{3} \int \frac{\mathrm{d} F_{1}}{\mathrm{~d} T_{2}}\left[U_{\mathrm{E}}\left(\boldsymbol{X}_{1}\right)+\frac{1}{2} \boldsymbol{V}_{1}^{2}\right] \mathrm{d} \tau_{2} \\
& =-\epsilon^{2} \int\left[U_{\mathrm{E}}\left(\boldsymbol{X}_{1}\right)+\frac{1}{2} \boldsymbol{V}_{1}^{2}\right] \mathrm{d} \tau_{1}+\mathcal{O}\left(\epsilon^{4}\right),
\end{aligned}
$$

and

$$
\begin{gathered}
\epsilon^{3} \int \frac{\mathrm{d} F_{2}}{\mathrm{~d} T_{2}} \mathrm{~d} \tau_{2}=2 \epsilon^{3} G M_{\mathrm{E}} \ln \left[\frac{R\left(T_{1}\right)+\boldsymbol{X}\left(T_{1}\right) \cdot \hat{\boldsymbol{N}}}{R\left(T_{2}\right)+\boldsymbol{X}\left(T_{2}\right) \cdot \hat{\boldsymbol{N}}}\right]+\mathcal{O}\left(\epsilon^{4}\right) \\
=2 \epsilon^{3} G M_{\mathrm{E}} \ln \left[\begin{array}{l}
\frac{\left|\boldsymbol{X}_{1}\left(T_{1}\right)\right|+\left|\boldsymbol{X}_{2}\left(T_{2}\right)\right|+\mid \boldsymbol{X}_{1}\left(T_{1}\right)}{\left.\mid \boldsymbol{X}_{2}\right) \mid} \\
\left|\boldsymbol{X}_{1}\left(T_{1}\right)\right|+\left|\boldsymbol{X}_{2}\left(T_{2}\right)\right|-\mid \boldsymbol{X}_{1}\left(T_{1}\right) \\
-\boldsymbol{X}_{2}\left(T_{2}\right) \mid
\end{array}\right]+\mathcal{O}\left(\epsilon^{4}\right),
\end{gathered}
$$

where different forms for Shapiro time delay can also be found in Nelson (2011). By putting all of the three equations above into Equation (A1), we can finally obtain that

$$
\begin{gathered}
\tau_{1}-\tau_{2}=\epsilon\left|\boldsymbol{X}_{1}\left(T_{1}\right)-\boldsymbol{X}_{2}\left(T_{2}\right)\right| \\
-\epsilon^{2} \int_{\tau_{2}}^{\tau_{1}}\left\{U_{\mathrm{E}}\left[\boldsymbol{X}_{1}(\tau)\right]+\frac{1}{2} \boldsymbol{V}_{1}^{2}(\tau)\right\} \mathrm{d} \tau \\
+\epsilon^{2} \int_{\tau_{2}}^{\tau_{1}}\left\{U_{\mathrm{E}}\left[\boldsymbol{X}_{2}(\tau)\right]+\frac{1}{2} \boldsymbol{V}_{2}^{2}(\tau)\right\} \mathrm{d} \tau \\
+2 \epsilon^{3} G M_{\mathrm{E}} \ln \left[\frac{\left|\boldsymbol{X}_{1}\left(T_{1}\right)\right|+\left|\boldsymbol{X}_{2}\left(T_{2}\right)\right|+\left|\boldsymbol{X}_{1}\left(T_{1}\right)\right|}{\left|\boldsymbol{X}_{1}\left(T_{1}\right)\right|+\left|\boldsymbol{X}_{2}\left(T_{2}\right)\right|-\mid \boldsymbol{X}_{1}\left(T_{1}\right)}\right]+\mathcal{O}\left(\epsilon^{4}\right) . \\
-\boldsymbol{X}_{2}\left(T_{2}\right) \mid
\end{gathered}
$$

In fact, the difference between the proper time and coordinate time can be neglected in the terms with the orders of $\epsilon^{2}$ and $\epsilon^{3}$ in the above equation. 